\title{
HIERARCHICAL METHODS FOR LANDMINE DETECTION WITH WIDEBAND ELECTRO-MAGNETIC INDUCTION AND GROUND PENETRATING RADAR MULTI- SENSOR SYSTEMS
}

\author{
Seniha Esen Yuksel', Ganesan Ramachandran ${ }^{1}$, Paul Gader ${ }^{1}$, \\ Joseph Wilson ${ }^{1}$, Dominic Ho ${ }^{2}$, and Gyeongyong Heo ${ }^{1}$ \\ ${ }^{1}$ The Department of Computer and Information Science and Engineering, University of Florida \\ ${ }^{2}$ The Department of Electrical and Computer Engineering, University of Missouri-Columbia, Columbia
}

\begin{abstract}
A variety of algorithms are presented and employed in a hierarchical fashion to discriminate both Anti-Tank (AT) and Anti-Personnel (AP) landmines using data collected from Wideband Electro-Magnetic Induction (WEMI) and Ground Penetrating Radar (GPR) sensors mounted on a robotic platform. The two new algorithms for WEMI are based on the In-phase vs. Quadrature plot (the Argand diagram) of the complex measurement obtained at a single spatial location. The Angle Prototype Match method uses the sequence of angles as a feature vector. Prototypes are constructed from these feature vectors and used to assign mine confidence to a test sample. The Angle Model Based KNN method uses a two parameter model; where the parameters are fit to the In-phase and Quadrature data. For the GPR data, the Linear Prediction Processing and Spectral Features are calculated. All four features from WEMI and GPR are used in a Hierarchical Mixture of Experts model to increase the landmine detection rate. The EM algorithm is used to estimate the parameters of the hierarchical mixture. Instead of a two way mine/non-mine decision, the HME structure is trained to make a five way decision which aids in the detection of the low metal anti personnel mines.
\end{abstract}

Index Terms - Landmine Detection, Hierarchical Mixture of Experts, Argand Diagram, Expectation Maximization, Iteratively Reweighted Least Squares.

\section{INTRODUCTION}

Ground-penetrating radar (GPR) and Wideband ElectroMagnetic Induction (WEMI) are two main sensors used in the detection of landmines. WEMI is used to detect highly metallic objects; whereas GPR provides information on the objects with little or no metal content; and is used to compensate WEMI. In this paper, new algorithms based on angle prototype matching and angle based modeling have been introduced and used on the WEMI data. For GPR data, LPP and spectral features were calculated. The outputs of all four algorithms have been treated as features, and are combined using the Hierarchical Mixture of Experts (HME) method to discriminate AT and AP mines from metallic and non-metallic clutter of various sizes. The HME method involves a hierarchical decision structure that utilizes the strengths of each algorithm/sensor to determine a confidence that a mine is present at the current location. The hierarchical approach uses soft-computing methods to determine how much each algorithm is effective in a decision.

Instead of a two way mine/non-mine decision, the HME structure is trained to make a five way decision as:

class-1: High metal (HM) anti-tank and anti-personnel mines class-2: Low metal anti-tank (LMAT) mines

class-3: Low metal anti-personnel (LMAP) mines

class-4: Metallic clutter (high, medium, low metallic)

class-5: Non-metallic clutter and blanks.

With this 5-way decision approach, it is possible to train the network specifically for the low metal anti-personnel mines, which are the hardest to detect because of their low metallic content and small sizes. At the output of the HME, the first three classes are summed up to be the mine confidence, and the last two are summed up to be the clutter probability. Since they all sum up to one, the Receiver Operating Characteristics (ROC) curves were plotted based on the mine confidences.

The structure of the paper is as follows: section 2 describes two methods to extract the features from WEMI data; and section 3 describes the feature extraction methods from GPR. All four features are trained using a Hierarchical Mixture of Experts (HME) algorithm as described in Section 4. The HME is trained using an EM algorithm; and the iteratively reweighted least squares (IRLS) algorithm is used to solve the maximum likelihood problem. Section 5 describes the data collection; and the experimental results are presented in section 6 .

\section{FEATURE EXTRACTION FROM WIDEBAND ELECTRO-MAGNETIC INDUCTION (WEMI)}

Argand diagrams have been one of the widely used tools 
to study complex data. An Argand diagram is a plot of the In-phase component against the Quadrature-phase component; and it provides a consistent framework to identify specific mine types. The Argand plots of two different mines have been shown in Fig. 1. The boxed AP mines in Fig.1 (a) show a different signature than the circular AT mines in Fig.1 (b). These mine characteristics in the Argand plots can be exploited in landmine detection.
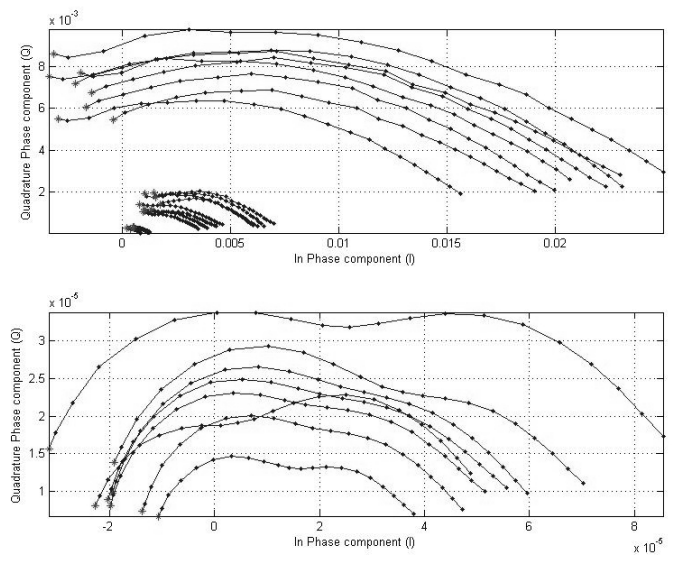

Fig. 1. Argand plots of WEMI response for a boxed AP mine and a circular AT mine at different locations and depths. Different points in the curve represent the WEMI response at different frequencies.

Although the signatures are similar in the above plots, there is variability in the amplitude and in the shift in real axis between different candidates of a same mine type. For that reason, a better way is to use the angles between the points in the Argand diagram for discrimination. As it is shown in Fig. 2, the variability between different candidates is now reduced as the angle is independent of amplitude and shift, two of the most difficult and unreliable [1] values to estimate.
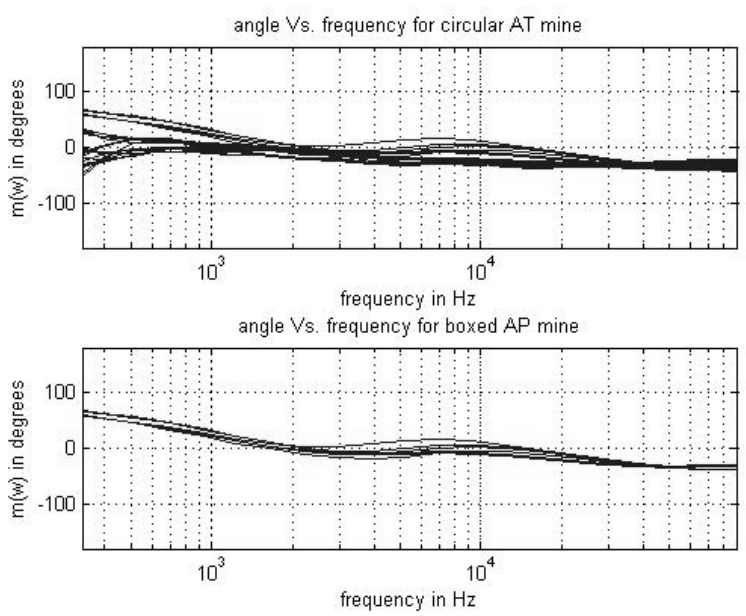

Fig. 2. Angle plots of WEMI response for a boxed AP mine and circular AT mine at different locations and depths.
The angles are calculated numerically by using a two-sided gradient from the Quadrature $Q$ and In-phase $I$ components as:

$$
\begin{aligned}
& \partial Q(\omega)=Q(\omega+\Delta)-Q(\omega-\Delta) \quad \partial I(\omega)=I(\omega+\Delta)-I(\omega-\Delta) \\
& m(\omega)=\operatorname{atan} 2(\partial Q(\omega), \partial I(\omega)) \\
& m=2 \tan ^{-1}\left(\frac{\partial Q(\omega)}{\partial I(\omega)+\sqrt{(\partial I(\omega))^{2}+(\partial Q(\omega))^{2}}}\right)
\end{aligned}
$$

\subsection{Angle Prototype Matching}

The angle sequences provide us with a valuable tool for discrimination as each object has its own signature. We use a point-wise median (median among candidates at a given frequency) as the prototype and compute the Euclidean distance as a goodness of fit measure.

$$
\begin{aligned}
& T_{i}=\{\text { all candidates of mine type } i\} \\
& K_{j}=\{\text { all candidates of nonmine type } j\} \\
& O=\left\{\bigcup_{i} T_{i}, \bigcup_{j} K_{j}\right\} \\
& M_{k}^{i}(\omega)=\left\{m_{k}(\omega): k \in T_{i}\right\} \\
& P_{i}(\omega)=\frac{\text { median }}{k}\left(M_{k}^{i}(\omega)\right) \\
& d_{j i}=\sum_{\omega}\left|m_{j}(\omega)-P_{i}(\omega)\right|, j \in O \\
& d_{j}^{*}=\frac{\min _{i}}{d_{j i}} \\
& \operatorname{conf}(j)=\frac{1}{1+e^{-\gamma\left(d_{j}^{*}-b\right)}}
\end{aligned}
$$

where,

$\gamma=$ rate parameter, and $b=$ bias parameter

\subsection{Angle Model Based KNN}

Miller et al. [2] proposed parametric models to model WEMI data; of which, the four parameter model in Eq. 1 was shown to be useful in landmine detection by Fails et al.[1]; where the parameters $\mathrm{A}, \mathrm{s}, \mathrm{c}$ and $\tau$ are restricted to be real.

$$
(I(\omega)+i Q(\omega))_{p 4}=A\left(s+\frac{(i \omega \tau)^{c}-2}{(i \omega \tau)^{c}+1}\right)
$$

Using the same approach as angle prototype matching, we modify this model to represent the angle between points in the Argand diagram as in Eq. 2. 


$$
m(\omega)=-2 \tan ^{-1}\left(\frac{\left((\omega \tau)^{2 c}-1\right) \sin \left(\frac{c \pi}{2}\right)}{\left[\begin{array}{l}
\left(1+(\omega \tau)^{2 c}\right) \cos \left(\frac{c \pi}{2}\right)+2(\omega \tau)^{c} \\
+\left|1+(\omega \tau)^{2 c}+2 \cos \left(\frac{c \pi}{2}\right)(\omega \tau)^{c}\right|
\end{array}\right]}\right)
$$

This gives us three features ( $\tau$, $\mathrm{c}$ and fitting error $\varepsilon$ ) which are then used for classification using a k-NN framework described by Fails et al.[1]. More details on our algorithm can be found in [3].

\section{FEATURE EXTRACTION FROM GROUND PENETRATING RADAR FROM GPR}

Linear Prediction Processing (LPP) features and Spectral features were extracted from Ground Penetrating Radar; and they were fused by the direct sum of the two (after proper scaling in LPP) divided by the square root of spectral compactness.

\subsection{Linear Prediction Processing}

Linear Prediction Processing (LPP) is a technique that removes the background component in the GPR data. It models the background using a dynamic linear predictor, where the current background vector sample is approximated by a linear combination of past background vector samples. The linear prediction coefficients are obtained by minimizing the mean-square prediction error, and they are different at different positions where different background vector samples are to be predicted. The LPP output is the difference between the current GPR vector sample and its predicted value generated from the linear prediction model, and it is the background removed data. The LPP feature is the maximum energy value of the background removed data around an alarm location declared by a pre-screener [4].

\subsection{Spectral Features}

Spectral feature is created by estimating the energy density spectrum (EDS) of an alarm at the declaration position and matching the estimated EDS with a template that is obtained from a landmine target. More than one template can be used, and in such a case the maximum of the matching score is assigned as the spectral feature. More details on the spectral features can be found in [5].

\section{HIERARCHICAL MIXTURE OF EXPERTS}

Hierarchical Mixtures of Experts is a tree structure introduced by Jacobs et al. [6] where each leaf of the tree is an expert (prediction) function, each node has a gating network that gives the prior probabilities; and the root is the final prediction. In our experiments, we used the 5-class output structure with depth being 2 , and hence there were five nodes for each gate as shown in Fig. 3.

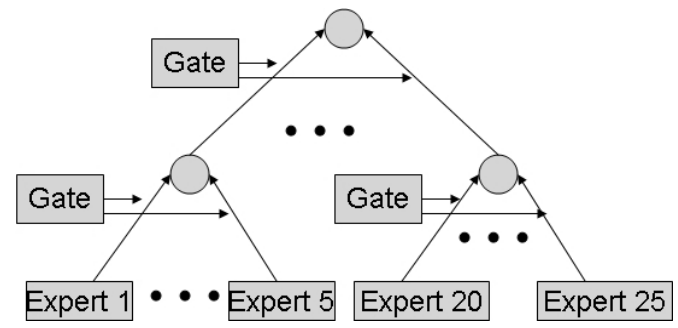

Figure 3: HME structure of depth 2, with 5 experts per each node.

For an $n$ dimensional data $x$; the outputs at the leaf nodes of the tree are given as $\mu_{i j}=f(U x)$ where $f$ is a fixed continuous nonlinearity and $U$ is a randomly initialized weight matrix [6].

At the non-leaf nodes, the gating network outputs $g_{i}$ are calculated as $g_{i}=\frac{\exp \left(\xi_{i}\right)}{\sum_{k} \exp \left(\xi_{k}\right)}$, where $\xi_{i}=v_{i}^{T} x$, and $v_{i}$ is a randomly initialized $n$ dimensional vector. Since the gating network outputs depend solely on the input, they are considered as prior probabilities.

The outputs at the non-leaf nodes of the tree are calculated as: $\mu=\sum_{i} g_{i} \mu_{i}$

For each expert, a true output $y$ is chosen from a distribution $P$ with mean $\mu_{i j}$; therefore, the total probability of generating $\mathrm{y}$ from $\mathrm{x}$ is given by

$$
P(y \mid x, \mu)=\sum_{i} g_{i} \sum_{j} g_{j l i} P\left(y \mid x, \mu_{i j}\right)
$$

where $j / i$ indicates $j^{\text {th }}$ unit in the $i^{\text {th }}$ gating network; so it is just an index to the hierarchy.

Based on this probability, Expectation Maximization has been used to adjust the weighting parameters that maximize the log likelihood. In the E-step, the posterior probabilities are computed from expert and gating outputs. In the M-step, the weights are updated to maximize the posterior probabilities. The problem turns into a generalized linear model, and the parameters can be updated using the iterative reweighted least squares (IRLS) algorithm [6]. 


\section{DATA COLLECTION}

The data from the experiments was collected in August 2007 from two geographically separated test sites that have different soil properties. Combined, both sites had a total of 11 high metal anti tank mines, 49 low metal anti tank mines, 30 high metal anti personnel mines, 66 low metal anti personnel mines; and 90 high metallic clutter objects; 28 medium metallic clutter objects; 28 low metallic clutter objects; 143 non-metallic clutter objects and blanks.

In each site, there were two collections in NW and SE directions. Both collections were combined; however, if an object was included in the training, it was included using both the directions (hence both data went into training); and neither was included in the testing. The experimental results presented in the next section were trained and tested in this way with a 10 -cross-fold validation.

\section{EXPERIMENTAL RESULTS}

The experimental results are presented using the Receiver Operating Characteristics (ROC) curves. The Receiver Operating Characteristics (ROC) curve specifies the probability of detection (PD) vs. the false alarm rate (FAR); and it is a common method to measure the performance of a mine detection system. The angle prototype match and angle model based KNN algorithms have been found to be very useful using the metal detector; giving 90/18 and 90/30 detection rates as shown in Fig.[1]. The fusion of the spectral features and the LPP features from the GPR gave a detection rate of 90/50. When the HME algorithm was used, the detection rates increased to $90 / 10$, outperforming the single use of all the algorithms. The HME training was fairly fast, and the testing is on the order of seconds. For the HME training, the network used was of depth 2, and using 5 experts.

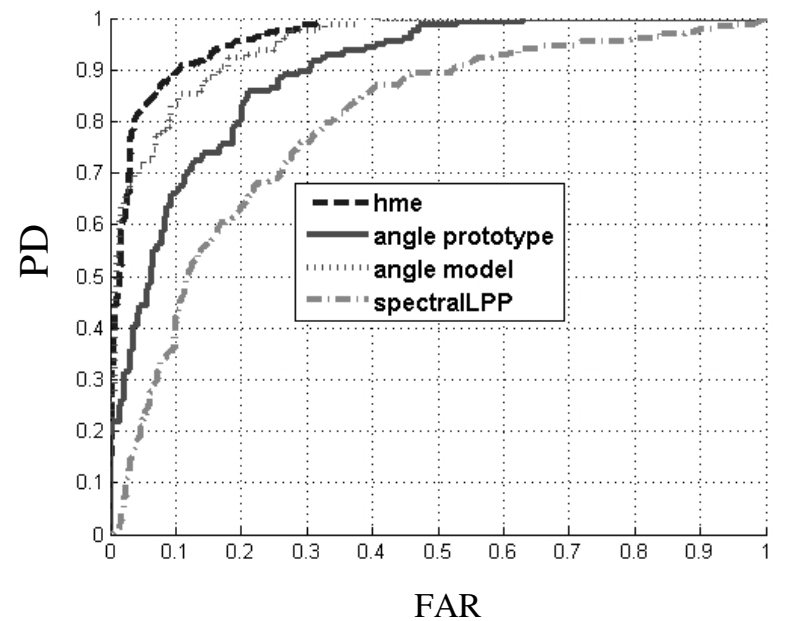

Figure 4: ROC curves from HME, Angle Prototype Match, Angle Model Based KNN, Spectral and LPP fusion. Using the other three features, HME was able to increase the detection rate to 90/10.

\section{CONCLUSION}

The HME acting on the four features calculated has been shown to generate good results. For the most part, specialization to learn the different kind of objects was accomplished by using a 5-class output in the HME. Our future work will include generating features for low metal anti personnel mines including anomaly detection; and specializing the training for these new features.

\section{ACKNOWLEGMENTS}

This research was supported by Army Research Office grant W911NF0510067.

\section{REFERENCES}

[1] E. Fails, P. Torrione, W. Scott, L. Collins, "Performance of a Four Parameter Model for Landmine Signatures in Frequency Domain Wideband Electromagnetic Induction Detection Systems", Proc. SPIE Vol. 6553, Detection and Remediation Technologies for Mines and Minelike Targets XII; Russell S. Harmon, J. Thomas Broach, John H. Holloway, Jr.; Eds. 2007.

[2] J.T. Miller, T. H. Bell, J. Soukup and D. Keiswetter, "Simple phenomenological models for wideband frequency-domain electromagnetic induction," IEEE Transactions on Geoscience and Remote Sensing, v. 39, no. 6, Jun. 2001, pages 1294-1298.

[3] G. Ramachandran, P. Gader, and J.N. Wilson, "GRANMA: Gradient Angle Model-Based Algorithm on Wideband EMI data for Landmine Detection", In preparation.

[4] H. Frigui, K. C. Ho, P. Gader, "Real-time landmine detection with ground-penetrating radar using discriminative and adaptive hidden Markov models", EURASIP Journal on Applied Signal Processing, v. 2005 n.1, p.1867-1885, January 2005.

[5] K.C. Ho, L. Carin, P.D. Gader, J.N. Wilson, “An Investigation of Using the Spectral Characteristics From Ground Penetrating Radar for Landmine/Clutter Discrimination", IEEE Transactions on Geoscience and Remote Sensing, volume 46, issue 4, part 2, April 2008 pp. 1177 - 1191.

[6] M.I. Jordan, R.A. Jacobs, "Hierarchical Mixture of experts and the EM Algorithm”, NeurComp(6), 1994, pp. 181-214. 